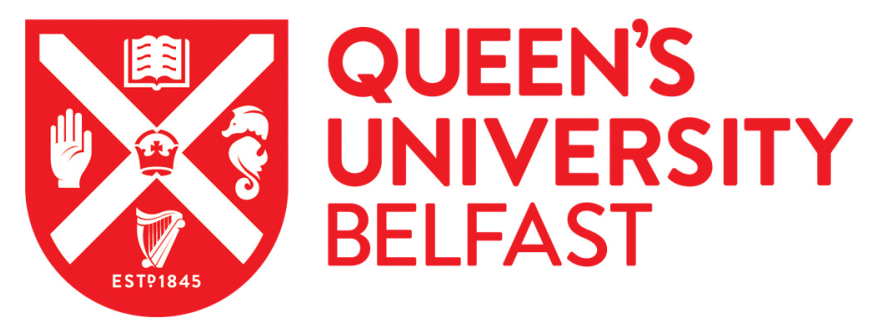

\title{
A promising laser nitriding method for the design of next generation orthopaedic implants: Cytotoxicity and antibacterial performance of titanium nitride (TiN) wear nano-particles, and enhanced wear properties of laser-nitrided Ti6Al4V surfaces
}

Chan, C. W., Quinn, J., Hussain, I., Carson, L., Smith, G. C., \& Lee, S. (2021). A promising laser nitriding method for the design of next generation orthopaedic implants: Cytotoxicity and antibacterial performance of titanium nitride (TiN) wear nano-particles, and enhanced wear properties of laser-nitrided Ti6Al4V surfaces. Surface and Coatings Technology, 405, [126714]. https://doi.org/10.1016/j.surfcoat.2020.126714

Published in:

Surface and Coatings Technology

Document Version:

Peer reviewed version

Queen's University Belfast - Research Portal:

Link to publication record in Queen's University Belfast Research Portal

\section{Publisher rights}

Copyright 2020 Elsevier.

This manuscript is distributed under a Creative Commons Attribution-NonCommercial-NoDerivs License

(https://creativecommons.org/licenses/by-nc-nd/4.0/), which permits distribution and reproduction for non-commercial purposes, provided the author and source are cited.

\section{General rights}

Copyright for the publications made accessible via the Queen's University Belfast Research Portal is retained by the author(s) and / or other copyright owners and it is a condition of accessing these publications that users recognise and abide by the legal requirements associated with these rights.

Take down policy

The Research Portal is Queen's institutional repository that provides access to Queen's research output. Every effort has been made to ensure that content in the Research Portal does not infringe any person's rights, or applicable UK laws. If you discover content in the Research Portal that you believe breaches copyright or violates any law, please contact openaccess@qub.ac.uk. 


\title{
A promising laser nitriding method for the design of next generation orthopaedic implants: Cytotoxicity and antibacterial performance of titanium nitride (TiN) wear nano-particles, and enhanced wear properties of laser-nitrided Ti6Al4V surfaces
}

\author{
${ }^{*}$ Chi-Wai Chan", James Quinn ${ }^{b}$, Issam Hussainc, Louise Carsonb, Graham C. Smith', \\ Seunghwan Lee
}

\author{
aSchool of Mechanical and Aerospace Engineering, Queen's University Belfast, BT9 5AH, UK \\ 'School of Pharmacy, Queen's University Belfast, BT9 7BL, UK \\ 'School of Life Sciences, University of Lincoln, Brayford Pool, Lincoln, LN6 7TS, UK \\ dFaculty of Science and Engineering, University of Chester, Thornton Science Park, Chester, CH2 4NU, UK \\ eDepartment of Mechanical Engineering, Technical University of Denmark, DK-2800, Kgs. Lyngby, Denmark
}

\begin{abstract}
In this study, fibre laser nitriding in open air was applied to the Ti6AI4V alloy in order to improve the wear resistance, thus minimising the generation of wear debris from the surfaces for load-bearing applications. The recent technological advancement to perform the laser nitriding process in open air allows the opportunity to surface-harden any curved and/or specific areas in the hip implants. The laser nitriding process was modulated between the pulsed mode and continuous wave (CW) mode by varying the duty cycle between $60 \%$ (pulsed) and $100 \%$ (CW). Our experimental investigations were divided into two stages in sequential order: Firstly, to create crack-free, homogenous and golden laser-nitrided surfaces by the proper selection of duty cycle. Secondly, it was to analyse the properties (both physical and chemical) of the wear debris as well as to evaluate their cytotoxicity and antibacterial performance. The laser-nitrided surfaces were characterised and tested using a variety of techniques, incl. optical microscopy, SEM-EDX, XRD, surface roughness and Vickers hardness measurements, as well as tribological tests (i.e. ball-on-disk wear tests and DLS). The wear debris from the laser-nitrided surfaces (collected in the wear tests) were analysed using TEM, XPS and SEM-EDX. Their toxicity was evaluated using in-vitro cell culture with macrophages at two time points ( $24 \mathrm{~h}$ and $48 \mathrm{~h}$ ). The antibacterial performance was tested in vitro against two of the most commonly implicated pathogens in orthopaedic infection, namely Staphylococcus aureus and Escherichia coli for $24 \mathrm{~h}$. Our findings indicated that the wear resistance of the surfaces after laser nitriding was significantly improved and the amount of wear debris generated was also significantly reduced. The wear particles from the laser-nitrided surfaces were in the nano-sized scale range $0.01 \mu \mathrm{m}$ to $0.04 \mu \mathrm{m}$ or $10 \mathrm{~nm}$ to $40 \mathrm{~nm}$ ). They were found to be less toxic towards RAW264.7 macrophages, yet display antimicrobial properties against Staphylococcus aureus, when compared with the larger particles (1.5 $\mu \mathrm{m}$ in size) from the untreated surfaces. It is envisioned that successful fabrication of the non-toxic and highly wear-resistant TiN layer in Ti6Al4V using the open-air laser nitriding technique can enable progress towards the development of metal-on-metal (MoM) hip implants fully made of Ti-based alloys.
\end{abstract}

Keywords: Laser nitriding, titanium nitride (TiN), nano-particles, orthopaedics, wear debris

*Corresponding Author: Chi-Wai Chan (c.w.chan@qub.ac.uk) 


\subsection{Introduction}

Orthopaedic hip implants are medical devices intended to restore the mobility and relieve pain in patients who suffer from arthritis and other hip associated diseases or injuries. An online report from the US Food and Drug Administration (FDA) indicates that the longevity of hip implants is related to a variety of factors such as (i) implant design features (size, shape and material), (ii) surgeon experience, and (iii) technique of implantation, as well as (iv) individual patient characteristics, including sex, age, weight, level of activity and concomitant health conditions [1]. Amongst these factors, damage to the implant materials caused by wear is the main challenge faced by all designs of hip implants, particularly for metal-on-metal (MoM) implants.

In the MoM design, both the ball and socket (or cup) components are made of metal. Rubbing between the metal femoral head and the metal socket (both of which usually made of CoCrMo) during walking or running can cause the release of metal ions and/or microscopic particulate matter [2]. Wear can also occur in other coupling components of the hip implant, namely the femoral head and the taper of a femoral stem in which micro-motions occur between the interior surface of the ball head and the tapered surface, causing the release of the metal ions and/or particles. Such metal ions and/or particles (if nano-sized in scale) can enter the bloodstream and be carried to, and accumulate in, different tissues or organs in the body. The MoM hip implants are suitable for young patients with more active lifestyle on account of their significantly lower wear rate (i.e. reduced by 60 times) than the conventional metal-on-plastics (MoP) design [3]. However, there is a trade-off between the reduced wear damage and the increased toxicity of the metal ions/particles being released.

Heavy metals are known to be health hazards and have adverse effects on cell growth and metabolism. Higher $\mathrm{Co}$ and $\mathrm{Cr}$ concentrations in blood have been associated with structural changes in neurological pathways, leading to neurological problems e.g. changes in brain structure and function [4]. Furthermore, accumulation of the metal wear particles can cause damage to the bones and/or soft tissues surrounding the hip implant. Such damage in the soft tissues is the main reason for pain and implant loosening, which can subsequently lead to device failure and revision surgery. The United Kingdom's (U.K.) Medicines and Healthcare products Regulatory Agency (MHRA) has issued a medical device alert including specific follow-up recommendations (including blood tests and imaging) for patients with MoM hip replacements [2]. Evidently, there is an urgent need to replace the CoCr-based metals in the MoM implant design.

Ti-based alloys, particularly Ti6Al4V (Ti Grade 5) have been successfully employed as implant materials for several decades given their excellent biocompatibility, mechanical and corrosion properties, as well as high strength to weight ratio. However, direct replacement of $\mathrm{CoCr}$ heads or sockets by the Ti6Al4V alloy is practically not possible given that the inferior wear resistance of Ti6Al4V can lead to the generation of a higher amount of potentially toxic wear particles. Exposure to high concentrations of $\mathrm{Al}$ and $\mathrm{V}$ ions can generate long/term health problems such as peripheral neuropathy, osteomalacia and Alzheimer's disease [5]. Therefore, Ti6Al4V can only be used as bearing surfaces with post surface hardening treatment.

Ti6Al4V can be surface hardened with titanium nitride (TiN) using physical, chemical or thermal treatment methods, e.g. physical or chemical vapour deposition (PVD or CVD), ion-implantation, laser surface nitriding, heat treatment to name a few. The problems of PVD or CVD concern the interface between the coating and substrate. It has been reported that third body wear can happen at the interface due to delamination of PVD-coated TiN on Ti6Al4V [6]. The coatings formed by ionimplantation are too thin (depth less than one micron) for long/term protection against wear. The hardening heat-treatment at elevated temperature can alter the whole structure of both the surface 
and bulk. In comparison, laser surface nitriding offers the unique advantage of creating a thick TiN layer (of up to $100 \mu \mathrm{m}$ ) which is metallurgically bonded with the substrate but without altering the bulk properties. The recent technological advancement to perform the laser nitriding process in open air allows the opportunity to surface-harden any curved and/or specific areas in the hip implants. The open-air laser nitriding has been successfully applied to commercially pure (cp) Ti, Ti6Al4V, NiTi and beta $\mathrm{Ti}$ alloys by the present authors to enhance the surface properties including wear, corrosion and antibacterial resistance $[7,8,9,10]$.

TiN is known to be a biologically inert material which shows promising blood tolerability properties (with a hemolysis percentage near to zero) [11]. TiN is often added as a coating layer to improve the tribocorrosion properties of Ti whilst maintaining biocompatibility [12]. TiN-coated commercially pure titanium (cp-Ti) implants have shown good cytocompatibility with mouse fibroblast cells [12]. Gordin et al. [13] demonstrated that TiN-coated $\mathrm{cp}-\mathrm{Ti}$ and Ti6Al4V show improved osteoblast proliferation compared to their controls. Furthermore, the antibacterial activity of TiN-coated Ti6Al4V was tested against five different strains of Streptococci and it proved to be bactericidal in vitro [14]. TiN-coated Ti plasma sprayed surfaces also demonstrated reduced bacterial adhesion and had a favourable biological affinity towards bone cell precursors [15]. The concept of applying laser nitriding technique to treat Ti6AI4V for orthopaedic applications has been proposed in the existing literature, and successful outcomes to achieve enhanced wear and corrosion properties by laser nitriding have been reported [7]. However, to the best of the authors' knowledge, practical usage of the laser-nitrided hip implants have not been realized yet given the potential cracking problems arising from (i) the brittle TiN surface layer and dendrites and (ii) the unknown toxicity of TiN wear particles to the surrounding cells and/or tissues. Until now, very little information about the toxicity of TiN particles has been available. In addition, the antibacterial performance of the TiN wear particles is not yet known.

In this study, there are two objectives to be achieved: Firstly, to form a wear-resistant, homogenous and crack-free TiN surface in Ti6Al4V by using the reported energy control method (i.e. laser-nitriding in modulated mode) [16]. Secondly, to evaluate the cytotoxicity and antibacterial performance of the TiN wear debris particles by in vitro cell culture with RAW264.7 murine macrophages at different time points (24h and $48 \mathrm{~h}$ ) as well as by in vitro evaluation of their antimicrobial properties against Staphylococcus aureus and Escherichia coli. In addition to the known benefits of TiN being highly wear and corrosion-resistant, we hypothesize that the wear particles from the laser-nitrided surfaces are less toxic and show enhanced antibacterial compared with those from the untreated surface. It is envisioned that successful fabrication of the non-toxic and highly wear-resistant TiN layer in the Ti6Al4V alloy using the open-air laser nitriding technique can enable progress towards the development of MoM hip implants fully made of Ti-based alloys. 


\subsection{Materials and Methods}

\subsection{Experiments and analysis in relation to the laser-nitrided surfaces}

\subsubsection{Materials}

The Ti-6Al-4V (in wt.\%) plate was sourced from American Elements (Los Angeles, California, United States) in the dimensions of $250 \mathrm{~mm} \times 250 \mathrm{~mm} \times 2 \mathrm{~mm}$. They were spark cut by wire electrical discharge machining (EDM) into the size of $125 \mathrm{~mm} \times 30 \mathrm{~mm}$ for laser nitriding. Before laser nitriding, the surfaces were ground and polished using a series of silicon carbide ( $\mathrm{SiC}$ ) sandpapers beginning with 120 grit until 1000 grit. The polished samples were cleaned and degreased in an ultrasonic water bath with acetone for $10 \mathrm{~min}$, followed with rinsing in deionised water and finally dried in a stream of cool air.

\subsubsection{Fibre laser nitriding in open air}

The samples were laser-nitrided in open air using the in-house SPI 200W fibre laser machine. The laser machine was integrated by Micro Laser systems BV (Driel, Gelderland, the Netherlands), and the fibre laser with the wavelength of $1064 \mathrm{~nm}$ was manufactured by SPI Lasers UK Ltd (South Hampton, Hampshire, UK). The laser machine can be modulated to $100 \mathrm{kHz}$ with the modulated pulses from $<5$ $\mu$ s to continuous wave (CW). The laser processing parameters were selected based on a set of preliminary experiments. They were set at $45 \mathrm{~W}$ (laser power), 60\% to 100\% (duty cycle, DC), $25 \mathrm{~mm} / \mathrm{s}$ (laser scanning speed), $1.5 \mathrm{~mm}$ (stand-off distance, SD), and purging with pure $\mathrm{N}_{2}$ gas at the gas pressure of 5 bar which was delivered coaxially with the laser beam. The laser spot size at the SD of $1.5 \mathrm{~mm}$ was calculated to be $100 \mu \mathrm{m}$. The overlapping ratio between adjacent tracks were $50 \%$. A detailed explanation for the concept of DC and the schematic of the experimental laser setup are reported elsewhere [16]. The laser-nitrided samples in this study were denoted as DC60, DC80 and DC100 (see Supplementary Information Fig. S1). The size of the nitrided areas was $10 \mathrm{~mm} \times 10 \mathrm{~mm}$, and each DC condition was repeated six times to ensure repeatability.

\subsubsection{Optical microscopy (OM) \& scanning electron microscope-energy-dispersive X-ray (SEM-EDX)}

The top and cross-sectioned surfaces after laser nitriding were imaged and analysed using a standard optical microscope (OM) and scanning electron microscope (SEM, FlexSEM 1000, Hitachi, UK). Standard metallurgical procedures were applied to prepare the cross-sectioned samples. The SEM was operated in the secondary-electron (SE) mode using a 5-kV beam. The compositions of the crosssectioned surfaces were analysed using EDX. The elemental compositions were measured and quantified in wt\%. The element distributions were determined using EDX mapping and line scans.

\subsubsection{X-ray diffraction (XRD)}

XRD data were acquired using a Bruker D8 Advance diffractometer (Bruker UK, Coventry) with Cu K $\alpha$ radiation, $\mathrm{Ni} \mathrm{K} \beta$ filter, fixed $0.6 \mathrm{~mm}$ divergence slit and fixed $9.5 \mathrm{~mm}$ anti-scatter slit. Data were scanned from $26^{\circ}$ to $98^{\circ}$ with $0.005^{\circ}$ increments. The XRD spectra were referenced to the International Centre for Diffraction Data (ICDD) PDF-4 database. Prior to measurement, the specimen surfaces were cleaned by ultrasonication in iso-propyl alcohol.

\subsubsection{Surface roughness measurements}

Surface roughness parameters: the average roughness of a surface ( $R a$ ) and the distance between the peak and valley in the surface $(\mathrm{Rz})$ were measured using a portable surface roughness tester (Mitutoyo Surftest). The Ra and Rz values were the average of five measurements taken in the two directions, namely (i) in parallel and (ii) perpendicular to the moving direction of the laser beam. 


\subsubsection{Vickers micro-hardness measurements}

The Vickers hardness tester (Future-Tech Corp FM-700, Japan) was used to measure the microhardness in the cross-sections. A load of $10 \mathrm{kgf}$ with a dwell time of $10 \mathrm{~s}$ was applied at each indentation. Five indentations in a row, with a separating distance of $100 \mu \mathrm{m}$, were made in each location including laser-nitrided zone (LNZ), heat-affected zone (HAZ), and base metal (BM). The indentations were located at the mean depth of LNZ and HAZ. The average of the results was used to represent the hardness in each location.

\subsubsection{Tribological tests and preparation of wear particles}

Ball-on-disk tribometry (CMS Instruments SA, Peseux, Switzerland) was carried out to apply tribostress to the samples. A loaded contact ( $10 \mathrm{~N})$ was made by a sapphire ball $(6 \mathrm{~mm}$ in diameter) on untreated or nitrided Ti samples. The sliding contact was made in a circular mode, and the linear sliding speed was $50 \mathrm{~mm} / \mathrm{s}$. As the primary purpose of the tribological tests was to generate wear particles to be used for cytotoxicity tests and antimicrobial analyses, all the tests were carried out in ultra-pure water (resistivity $>18 \mathrm{M} \Omega$ ) to avoid any coating of particles with biological substances. The fluid containing wear particles was collected using a micropipette in three intervals in terms of sliding distance: 0 to $200 \mathrm{~m}, 200$ to $500 \mathrm{~m}$, and 500 to $1,000 \mathrm{~m}$. At each interval the fluid was collected, and the tribo-cup was dried up by blowing $\mathrm{N}_{2}$. Then, a new batch of water $(3 \mathrm{ml})$ was added for the next interval experiment. As the first characterization of wear particle size distribution, dynamic light scattering (DLS, Zetasizer ZSP model, Malvern Instruments Ltd, Worcestershire, UK) was performed on each fluid containing wear debris generated from different samples with varying DC and sliding contact intervals. DLS analysis was performed individually on the fluid samples collected at each interval. Meanwhile, the fluids collected from the sliding distances of 200 to $500 \mathrm{~m}$ and 500 to 1,000 m were added together for further analysis (TEM, XPS, SEM-EDX) and cytotoxicity tests, due to visibly smaller amount of wear particles in those samples after centrifugation (15 $\mathrm{min}$ at 6,000 rpm). Thus, the samples were denoted as ' $200 \mathrm{~m}$ ' or ' $+800 \mathrm{~m}$ ' to refer to the first sample $(0$ to $200 \mathrm{~m})$ and the addition of the second and the third (200 to $500 \mathrm{~m}$ and 500 to $1,000 \mathrm{~m}$ ) thereafter. Lastly, wear volume was characterized by a profilometer (Alicona, Brucker) after the entire sliding contact of 1,000 m.

\subsection{Experiments and analysis in relation to the wear particles}

\subsubsection{Transmission electron microscopy (TEM)}

The wear particle suspensions were pipetted onto holey carbon film TEM grids to disperse the particles, followed by drying in air on Whatman No 1 filter papers. The grids were imaged in a Phillips CM20 TEM under bright field conditions using a $200 \mathrm{kV}$ primary beam. Data were recorded using a Gatan Inc. (Pleasanton, CA) type 782 camera at $1350 \times 1040$ pixel resolution and processed using Gatan Digital Micrograph v3 software.

\subsubsection{X-ray photoelectron spectroscopy (XPS)}

Samples of the wear particles were prepared for XPS by drying several drops of the wear particle suspensions onto clean gold film. Data were acquired with monochromatic Al Ka radiation using a bespoke UHV system equipped with Specs GmbH (Berlin) PHOIBOS 150 analyser and FOCUS500 monochromator. Survey scans were made at $50 \mathrm{eV}$ pass energy; higher resolution scans over individual photoelectron lines made at $20 \mathrm{eV}$ pass energy. Data processing was carried out using CasaXPS v2.3.16. 


\subsubsection{SEM-EDX}

The samples prepared for XPS were subsequently also examined by SEM and analysed by EDX using a Zeiss 1455VP SEM (Cambridge, UK) equipped with Oxford Instruments X-act X-ray detector and INCA software (Oxford, UK).

\subsubsection{Cell culture and wear particle analysis}

All cell culture procedures were performed under sterile conditions within a Class II Microbiological Safety Cabinet. The murine macrophage cell line, RAW 264.7, was purchased from American Type Culture Collection (ATCC). Macrophages at passage 5 were grown in tissue culture medium consisting of Dulbecco's Modified Eagles Media (DMEM) (with L-glutamine) (Sigma Aldrich, Ltd.), supplemented with $10 \%$ foetal calf serum (FCS) (Sigma Aldrich, Ltd.), and $1 \%$ of penicillin/streptomycin (Sigma Aldrich, Ltd.), and placed in an incubator set at $37^{\circ} \mathrm{C}, 5 \%$ humidified $\mathrm{CO}_{2}$ (Wolf Laboratories, Ltd.).

Wear particles were recovered by centrifugation, and the particle pellet was weighted, UV sterilized for one hour, and resuspended by vigorous vortex mixing, and diluted at a concentration of $10 \mu \mathrm{g} / \mathrm{mL}$ to $50 \mu \mathrm{g} / \mathrm{ml}$ in complete Dulbecco's modified Eagle medium. The cells to be used for MTT assay were cultured in T-75 tissue culture flask (Corning). When cells reached a high level of confluence $(80 \%-$ $85 \%$ sub-confluent), the cells were detached from the flask surface via the use of $0.25 \%$ trypsin and $0.02 \%$ EDTA solution. Cells were then re-suspended and seeded on 96 -well culture plates at 10,000 cells $/ \mathrm{mL}$ cell in a complete cell culture medium. After $24 \mathrm{~h}$ in culture, cell media was removed and replaced by fresh complete cell culture medium containing the following concentrations of the wear particles: $10,20,30,40,50 \mu \mathrm{g} / \mathrm{mL}$. Cell cultures were maintained for $24 \mathrm{~h}$ and $48 \mathrm{~h}$ in a cell culture incubator at $37^{\circ} \mathrm{C}$ and $5 \% \mathrm{CO}_{2}$.

3-(4,5-dimethylthiazol-2-yl)-2,5-diphenyltetrazolium bromide (MTT) solution was used to evaluate the effect of different concentrations of wear particles on mitochondrial activity of macrophages cells. 50 $\mu \mathrm{l}$ was added to the 96 well plates at $24 \mathrm{~h}$ and $48 \mathrm{~h}$. Cells were incubated with MTT for approximately $5 \mathrm{~h}$ to allow metabolism to insoluble formazan. The MTT-media solution was aspirated and $100 \mu \mathrm{l}$ of Dimethyl sulfoxide (DMSO) added to wells to dissolve the purple formazan product. Absorbance was read at $570 \mathrm{~nm}$ by a BioRad imark microplate reader. All experiments were carried out in triplicate and the values were presented as a percentage absorbance for treated cells against the untreated control.

\subsubsection{Antibacterial tests and wear particle analysis}

The wear debris particles were generated following the methodology described previously in Section 2.1.7., and were derived from either the laser-nitrided surface, or the untreated surface of Ti6Al4V. E. coli (ATCC 11303) and S. aureus (ATCC 6538) were cultured in Müller-Hinton Broth (MHB; Oxoid) for $18 \mathrm{~h}$ at $37^{\circ} \mathrm{C}$ on a $100 \mathrm{rpm}$ gyrorotatory incubator, following which the bacterial culture was adjusted to an optical density of 0.3 at $550 \mathrm{~nm}$ and diluted 1 in 50 with MHB, resulting in a bacterial inoculum of $\sim 10^{6} \mathrm{CFU} / \mathrm{mL}$.

Both the laser-nitrided and untreated Ti6AI4V wear particles were suspended in phosphate buffered saline (PBS) at a concentration of $20 \mu \mathrm{g} / \mathrm{mL}$. Vigorous vortex mixing and sonication was used to ensure homogenous distribution of the wear particles throughout the suspension.

This suspension was added to the MHB bacterial inoculum in an equi-volume ratio, so that finally the working concentration of wear particles was $10 \mu \mathrm{g} / \mathrm{mL}$. Each of the samples was tested in triplicate, and a blank control consisted of the sterile PBS vehicle was used. The samples were then incubated for $24 \mathrm{~h}$ in the dark in a 100 -rpm gyrorotatory incubator at $37^{\circ} \mathrm{C}$. Following this, the Miles and Misra 
method was utilised to determine the number of colony forming units of each bacteria species per millilitre (CFU/mL) [17]. 


\subsection{Results and Discussion}

\subsection{Characteristics of untreated and laser-nitrided surfaces}

\subsubsection{OM morphology and microstructure}

Fig. 1(a-c) shows the optical micrographs (OM) of the top and cross-sectioned surfaces in the Ti6Al4V samples nitrided at different duty cycles. Fig. 1(d) depicts the depth of laser-nitrided zones (LNZ) measured from Fig. 1(a-c) using ImageJ software $(n=8)$.

As seen in Fig. 1(a-c), the sample surfaces were fully covered by laser tracks and exhibited gold colour, which is the main characteristic of titanium nitride (TiN). When compared with DC60, discolouration occurred in the surfaces of DC80 and DC100. The discolouration, as reflected by the appearance of clusters in light blue colour, was found to be distributed preferentially at the perimeter of the laser tracks. The discolouration was more significant in DC100 than DC80. It has been previously reported that the discolouration is due to the oxygen $(0)$ intrusion from the surrounding air during the openair laser nitriding process [8]. At higher duty cycles, the laser energy input was higher because the laser-material-gas interaction time was longer, allowing a higher amount of energy and longer time for $\mathrm{O}$ to react with the molten materials in the surfaces, thus leading to more serious contamination of $O$ in the surfaces.

Likewise, the higher energy input together with the longer interaction time, when treating the samples at higher duty cycles, resulted in wider track width and deeper LNZ. The track width increased from $0.11 \mathrm{~mm}$ to $0.17 \mathrm{~mm}$ whilst the LNZ depth increased from $41 \mu \mathrm{m}$ to $205 \mu \mathrm{m}$ when increasing the duty cycle from $60 \%$ to $100 \%$.

When looking at the cross-sectioned OM images in higher magnification, two types of microstructure: (i) dendrites and (ii) mushy zone can be seen in the LNZ. The formation mechanisms of the mushy zone, which is formed due to the stagnation effect of growing dendrites in the melt pools, has been reported elsewhere [18]. It is interesting to note that the LNZ of DC60 and DC80 tended to be dominated by the mushy zone whilst the majority of LNZ in DC100 was occupied by the fully-grown dendrites.

The shape of the melt pools was found to be irregular at the boundary line between LNZ and HAZ. Furthermore, cracks with a recognizable size were identified in the cross-sectioned LNZ in DC80 and DC100. In contrast, the sample nitrided at DC60 was completely absent from cracks. The irregularlyshaped boundary line is caused by the local melting at the boundary area due to the exothermic reactions in forming the TiN dendrites. It is known that the formation of cracks in laser nitriding of Ti alloys is due to excessive build-up of residual tensile stress in the solidified melt pools. The magnitude of residual stress in the solidified melt pools is related to the size and population of dendrites as well as the overlapping ratio between the laser tracks. To minimise crack formation, these dendrite-related factors have to be carefully controlled and minimised, which can be done by reducing the laser energy input and interaction time during laser nitriding.

\subsubsection{SEM-EDX elemental composition analysis}

Fig. 2(a-c) show the results of SEM-EDX analysis for the laser-nitrided samples of DC60, DC80 and DC100 in their cross-sections. The SEM-EDX results included the three different modes of scans, namely, the elemental (i) mapping, (ii) line and (iii) area scanning profiles. The composition in the BM area in each sample was used as a control. 
A general trend, which can be observed from the composition spectra of all laser-nitrided samples in Fig. 2 , is that the percentage of the metal elements, namely $\mathrm{Ti}, \mathrm{Al}$ and $\mathrm{V}$, was reduced but that of the interstitial element $\mathrm{O}$ increased after laser nitriding. The reduction in the Ti and $\mathrm{Al}$ contents was found to be more significant when compared with $\mathrm{V}$ in the laser-nitrided samples. With regards to the enrichment of $\mathrm{O}$, it tended to be more significant with increased duty cycles. On the other hand, the results in the mapping scans indicate that the LNZ of all nitrided samples was obviously depleted of Al whereas the $\mathrm{Ti}$ and $\mathrm{V}$ contents were retained and were fairly evenly distributed in the LNZ. The line profile results agreed with the mapping results in that the content of Al dropped significantly in the LNZ in all the nitrided samples; however the Al content in the HAZ returned to the amount similar to the BM. The fluctuations of Al content in the LNZ, as denoted by the occurrence of peaks and troughs in the line scan profiles in Fig. 2, indicate that the Al content was distributed heterogeneously in the LNZ. Such fluctuations were more serious in the samples nitrided at DC80 and DC100 compared with DC60.

The reduction of metal fractions in the LNZ is mainly attributed to the enrichment of $O$ in the LNZ. Since the laser nitriding process was conducted in the open-air condition, the $\mathrm{O}$ in the surrounding air could intrude into the melt pool by the turbulent current which was generated when the high pressure $\mathrm{N}_{2}$ gas impinged on the material surface. After entering into the melt pool, some $\mathrm{O}$ was transported from the surface to the deeper location of the melt pool by (i) diffusion at elevated temperature and (ii) Marangoni convection flow. The finding in the authors' previous research [8] indicates that the laser-gas-material interactions, as reflected by the plasma brightness and presence of sparking in the plasma, were stronger when the Ti6AI4V was nitrided in a higher power regime. It is believed that stronger laser-gas-material interactions could enhance the turbulent current, bringing more $\mathrm{O}$ into the melt pool. It can also increase the local temperature of the melt pool and thus result in a stronger Marangoni convection effect. The synergistic effects of stronger turbulent flow, higher local temperature and stronger Marangoni current are the main contributors to cause a higher enrichment of $O$ in the LNZ when the samples were nitrided at higher duty cycles (DC80 and DC100).

Besides, the depletion and inhomogeneous distribution of Al in the LNZ can be explained by the following two reasons: (i) metallurgical segregation due to rapid solidification and (ii) vaporisation of $\mathrm{Al}$, provided that the surface temperature in the melt pool can be raised up to several thousand degrees in the laser nitriding process. Since the boiling point of $\mathrm{Al}$ is the lowest $\left(2470^{\circ} \mathrm{C}\right)$ compared with $\mathrm{Ti}\left(3287^{\circ} \mathrm{C}\right)$ and $\mathrm{V}\left(3407^{\circ} \mathrm{C}\right)$, the probability for the occurrence of Al vapourisation in the melt pool, particularly in the region near the surface, is higher than Ti and Al.

It is worthwhile to note that the results of EDX analysis showed no N in the LNZ of DC60 and DC100 samples. This could be probably due to a problematic overlap of Ti peak (L-line) with $\mathrm{N}$ peak (K-line) in the EDX spectrum.

\subsubsection{Roughness measurements (in microscale)}

Surface roughness is an important measure to determine the surface quality of a load-bearing implant as it can directly influence how the bearing surfaces interact with each other when two of them come into contact. It can also influence the initial adherence of living organisms (e.g. human cells and bacteria) when the implant contacts with the ubiquitous interstitial fluid. It is known that living organisms react differently with roughness in different scales, namely nano- and micro-sized scales. Roughness in the microscale dictates how the cells attach, align and organise with surface features, whereas roughness in the nanoscale controls the protein interactions of cells [19]. The roughness scale being investigated in this study was in the micro-scale. Fig. 3(a-b) provides the results of roughness measurement for the laser-nitrided samples in terms of (a) average $R a$ and (b) average $R z(n=5)$. The 
roughness of untreated BM was used as control. The roughness measurement was conducted in two different orientations: parallel and perpendicular to the laser tracks.

As seen in Fig. 3, the Ra of the laser-nitrided surfaces measured in the parallel and perpendicular directions were between $2.6 \mu \mathrm{m}$ and $4.1 \mu \mathrm{m}$ and between $2.7 \mu \mathrm{m}$ and $4.6 \mu \mathrm{m}$, respectively. The Rz of the nitrided surfaces in the parallel direction were between $14.0 \mu \mathrm{m}$ and $22.1 \mu \mathrm{m}$ whilst those in the perpendicular direction were between $15.6 \mu \mathrm{m}$ and $24.3 \mu \mathrm{m}$. The Ra of $\mathrm{BM}$, in the parallel and perpendicular directions, were $0.15 \mu \mathrm{m}$ and $0.26 \mu \mathrm{m}$ respectively. The $\mathrm{Rz}$ of $\mathrm{BM}$ in the parallel and perpendicular directions were $1.2 \mu \mathrm{m}$ and $2.3 \mu \mathrm{m}$.

The $\mathrm{Ra}$ and $\mathrm{Rz}$ measured in the perpendicular direction were in general higher than those measured in the parallel direction. All surfaces after laser nitriding were significantly roughened, with the surfaces nitrided at higher duty cycles (DC 80 and DC100) showing a higher Ra value. The Ra of the laser-nitrided surfaces was at least 10 times higher than the Ra of BM. A similar observation can be found in the result of Rz. Nitriding the surfaces at higher duty cycles resulted in a higher Rz value. The $\mathrm{Rz}$ of the nitrided surfaces was at least 6 times higher than the Rz of BM. When comparing between the results of $\mathrm{Ra}$ and $\mathrm{Rz}$ (including both laser-nitrided surfaces and BM), the Rz was obviously higher than the Ra.

$\mathrm{Ra}$ is a roughness parameter to measure the average vertical distance of all peaks and valleys in the roughness profile. In the measurement of Ra, occasional outlying points are neutralised and thus the extreme points in the profile have an insignificant impact on the final value of $R a$. In contrast, $R z$ measures the average distance of the five highest peaks and the five lowest valleys in the roughness profile. Existence of the extreme points in the profile can be reflected by the final value of Rz. Therefore, $\mathrm{Ra}$ and $\mathrm{Rz}$ have to be considered together in order to truly describe the surface roughness conditions. The obviously higher $\mathrm{Rz}$ compared with the Ra indicates that the surface roughness of the laser-nitrided samples were not homogenous across the entire surface.

The difference between the roughness measurements made in the two different orientations is due to the presence of features in different scales in the nitrided surfaces. In the parallel direction, the measurement was made along the laser tracks inside which only micro-features e.g. ripples and lamellae were present. However, in the perpendicular direction, the stylus tip moved across the laser tracks. Since the laser tracks overlapped with each other, a high number of boundaries between the overlapped laser tracks existed. The boundary between the overlapped tracks had a higher peak-tovalley distance than the aforementioned micro-features. Therefore, the Ra and Rz values measured in the perpendicular direction were higher than those in the parallel direction. The surface roughening mechanisms due to laser nitriding has been reported elsewhere [9].

\subsubsection{XRD phase structure characterisation}

The XRD diffraction patterns for the laser-nitrided samples between 30 and 50 degrees 2-theta are shown in Fig. 4. BM was used as a reference. The XRD results showed the existence of $\alpha-\mathrm{Ti}, \beta-\mathrm{Ti}$ and TiN in the nitrided surfaces, whilst only $\alpha$-Ti was present in the BM surface. Broadly, the XRD patterns for the nitrided surfaces showed the $\alpha$-Ti peaks reduced in intensity whilst the TiN peaks increased through the series of DC60 to DC100. The surface layers created after laser nitriding were not purely composed of TiN. A small number of phases from the parent metals was still present. The ratio between TiN and the phases from the parent metals was determined by the laser energy input and laser-material-gas interaction time, which were controlled by changing the duty cycle in this study. With higher duty cycles, the higher energy input together with longer interaction time leads to the 
formation of a surface layer with a higher fraction of TiN. This is due to the complete conversion of parent Ti phases into TiN in the nitriding process.

\subsubsection{Vickers micro-hardness measurements}

Fig. 5 shows the results of Vickers hardness measurement for the laser-nitrided samples at different locations in the cross-section (LNZ, HAZ and BM). As observed in Fig. 5, the hardness in LNZ was the highest and lay between $795 \mathrm{HV}$ and $844 \mathrm{HV}$. Compared with LNZ, reduced hardness was found in HAZ. The hardness of HAZ was between $448 \mathrm{HV}$ and $585 \mathrm{HV}$. BM had the lowest hardness of $374 \mathrm{HV}$. The hardening effect in LNZ is due to the formation of TiN dendrites/mushy zone. It is interesting to note that the hardness of LNZ in the samples nitrided between DC60 and DC100 was similar, indicating the mushy zone (i.e. dendrites in their growing form) and the fully-grown TiN dendrites possessed a similar hardness to each other. When comparing with DC60 and DC80, the LNZ in DC100 showed a higher variation in the hardness, as indicated by the longer error bar associated with the hardness result of DC100. This means that the laser nitriding process conducted at DC60 and DC80 was more stable than that at DC100, yielding a LNZ with a higher hardness homogeneity throughout the surface. The hardening effect in HAZ can be explained by solid state diffusion of $N$ in the substrate at elevated temperature.

\subsubsection{Tribological tests and wear particles}

Fig. 6(a-c) show the results of tribological tests for the laser-nitrided samples of DC60 to DC100. Fig. $6(a-b)$ provide the size distribution of wear particles measured by DLS technique in terms of (a) intensity (\%) vs. Hydrodynamic diameter $(\mathrm{nm})$ and $(\mathrm{b})$ Z-average $(\mathrm{nm})$. The wear particles collected at three intervals of sliding distance: 0 to $200 \mathrm{~m}, 200$ to $500 \mathrm{~m}$ and 500 to $1000 \mathrm{~m}$ were analysed and compared. Fig. 6(c) gives the results of volume loss (\%) in different laser-nitrided samples after completing the wear tests at $1000 \mathrm{~m}$. The results for untreated Ti6Al4V sample are shown in Fig. S2 in Supplementary Information. Briefly, the size profiles of untreated Ti6Al4V sample are much larger than those of DC60 to DC100 shown in Fig. 6(a), and it indicates the substantial reduction of wear of Ti6Al4V samples upon surface nitriding.

It should be noted that when the size profiles of wear particles from laser-nitrided Ti samples were plotted in terms of volume (\%), a single dominant peak was shown with the maximum at 7-8 $\mathrm{nm}$ and a trace of larger peaks (Supplementary Information Fig. S3). More importantly, they were virtually indistinguishable from each other for all the samples and sliding distance intervals. This means that the portion (\%) of larger wear particles (ca. 20 to 2,000 nm) presented in Fig. 6(a) should be, in fact, very little in numbers. Nevertheless, the intensity plots in Fig $6(\mathrm{a})$ display interesting trends due to high sensitivity of DLS towards large particles. Firstly, the influence of accumulated sliding contacts is increasingly more significant for the samples with higher duty cycle. For instance, the overall size distribution profiles of the DC60 samples remain nearly the same over the three intervals. In contrast, the size profiles for DC80 and DC100, especially those in large diameter (20 - 2,000 nm), are significantly varied as a function of sliding distance intervals. Secondly, the comparison of the size profiles within 0 to $200 \mathrm{~m}$ interval shows a gradual increase of larger shoulder peak in weight (indicated by arrows) with increasing duty cycle. This interval is particularly important as the wear debris in this interval is likely to have originated predominantly from the topmost LNZ layer. As the accumulated sliding distance is increasing, i.e. in the 200 to $500 \mathrm{~m}$ interval and in the 500 to $1000 \mathrm{~m}$ interval, the difference in size profiles is much smaller across samples with varying duty cycle. Overall, the size distribution profiles in Fig. 6(a) reveal that the weight of larger particle tends to increase with increasing duty cycle, especially those generated from the topmost LNZ layer. 
This trend is more clearly visualized in Fig. 6(b), where the Z-averages of the particle size from the samples over the sliding distance intervals are presented. The variation of Z-average along with the progress in sliding distance appears to be smaller for DC60 samples compared to DC80 and DC100, especially due to the drastic variation in the first sliding distance interval of 0 to $200 \mathrm{~m}$ to the next 200 to $500 \mathrm{~m}$ for DC80 and DC100 samples. The comparison in the 0 to $200 \mathrm{~m}$ intervals shows that the Zaverages of DC80 (ca. $74 \mathrm{~nm}$ ) and DC100 (ca. $67 \mathrm{~nm}$ ) are significantly larger than that of DC60 (ca. 38 $\mathrm{nm}$ ), whereas those acquired from the interval of 200 to $500 \mathrm{~m}$ and 500 to $1000 \mathrm{~m}$ are nearly the same for all three samples. Thus, the consistent profiles of size distribution and the small difference in Zaverages across the three intervals in the DC60 samples indicate that the LNZ in DC60 exhibited a more homogeneous wear resistance along with its depth. Meanwhile, the wear resistance of LNZ in DC80 and DC100 was varying along with the depth with the region near the top surface possessing more inferior wear resistance than the inner regions of LNZ. As a reference, the Z-average values for untreated Ti6Al4V samples were $0.88 \mu \mathrm{m}, 0.87 \mu \mathrm{m}$, and $1.39 \mu \mathrm{m}$ for 0 to $200 \mathrm{~m}, 200$ to $500 \mathrm{~m}$, and 500 to $1,000 \mathrm{~m}$, respectively, which are more than an order of magnitude larger those of nitrided Ti6Al4V samples.

The volume loss data $\left(\mathrm{mm}^{3}\right)$ in Fig. $6(\mathrm{c})$ were acquired after $1,000 \mathrm{~m}$ total sliding distance by profilometry. The results show that DC60 had the least amount of wear damage $\left(0.055 \mathrm{~mm}^{3}\right)$ compared with those in DC80 and DC100 which behaved similarly to each other $\left(0.070 \mathrm{~mm}^{3}\right)$. The superior wear resistance of the sample nitrided at lower (DC60) than higher duty cycles (DC80 and 100 ) is attributable to the quality of LNZ which can be judged by the (i) microstructural homogeneity and (ii) cracking formation. The samples nitrided at the higher duty cycles tend to have a gradient structure in the LNZ (in Fig. 1(b-c)) which is a mix of mushy zone and dendrites in DC80 or coarse (region near the top surface) and fine (inner regions) dendrites in DC100. In contrast, DC60 possessed a more homogeneous structure comprising mainly of mushy zones in the LNZ. Furthermore, the lower wear resistance of DC80 and DC100 than DC60 can be explained by the presence of cracks in the LNZ. All these findings point to the fact that the nitrides formed at higher duty cycles were more brittle compared with that treated at a lower duty cycle. The influence of increased surface roughness and inhomogeneity as well as the inclusion of parent material in the nitride layer on the tribological properties have been reported elsewhere [20].

\subsection{Characteristics of the wear particles from untreated and laser-nitrided samples}

\subsubsection{TEM analysis for the $\mathrm{BM}$ and TiN wear particles}

Fig. 7(a-b) shows the TEM micrographs of wear particles collected from the untreated (BM) and lasernitrided (DC100) samples after undergoing the wear tests at the sliding distance of $200 \mathrm{~m}$ and $+800 \mathrm{~m}$ (i.e. 200 to $500 \mathrm{~m}$ and 500 to $1,000 \mathrm{~m}$ ), respectively. The particle size and aspect ratio of the wear particles were measured using the ImageJ software and the results are shown in Fig. 8(a-b). The TEM images used for determining the particle size and aspect ratio are given in Supplementary Information (Fig. S4 and S5).

As seen in Fig. 7(a), the wear particles in the BM samples collected at $200 \mathrm{~m}$ and $+800 \mathrm{~m}$ were mainly composed of particles in the micro-and submicron-sized scales. These large particles were irregular and elongated in shape. They were electron-opaque (i.e. electrons were not transmitted through them). In addition, three types of nano-particles were also present in the BM wear debris, identified as: primary particles, aggregates (clusters of chemically-bonded particles) and agglomerates (clusters of particles loosely bound together by Van der Waals force). In comparison, only particles in the nanosized range were observed in the laser-nitrided samples (DC100). As for the laser-nitrided samples, the nano-particles were present in the wear debris as primary particles, aggregates and agglomerates. 
However, the larger particles were only found in the BM samples, in both $200 \mathrm{~m}$ and $+800 \mathrm{~m}$, and were not found in the wear debris from the laser-nitrided samples.

The average size of wear particles in the BM samples at $200 \mathrm{~m}$ and $+800 \mathrm{~m}$ were basically the same (i.e. measured to be $1.5 \mu \mathrm{m}$ and $1.4 \mu \mathrm{m}$, respectively). Nevertheless, their average aspect ratios were slightly different, with the particles collected at $200 \mathrm{~m}$ having an aspect ratio of 1.5 whilst those collected at $+800 \mathrm{~m}$ showed an average value of 1.7. Regarding the laser-nitrided samples, the particles collected at $200 \mathrm{~m}$ were found to be larger than those collected at $+800 \mathrm{~m}$ (average particles sizes of $0.04 \mu \mathrm{m}$ versus $0.01 \mu \mathrm{m}$ or $40 \mathrm{~nm}$ versus $10 \mathrm{~nm}$ ). This finding agreed well with the DLS results in the previous section. The average aspect ratio of the wear particles at the two distance were found to be the same, at 1.3, indicating that the particles in the wear debris of the laser-nitrided samples had an ellipsoid shape.

\subsubsection{SEM-EDX analysis for the BM and TiN wear particles}

Fig. 9(a-b) show the SEM images and the element distribution maps for different elements in the wear particles from BM and laser-nitrided samples. It is important to note that the samples were not in powder form but a suspension of wear debris particles in water. The samples being analysed in SEMEDX were either the dried powder (if the particle size was large enough to be captured by SEM) or in the form of a suspension (if the particle size was too small) dried onto a clean Au substrate.

For wear particles from the BM samples, the SEM image showed a particle-size of $1 \mu \mathrm{m}$ to $3 \mu \mathrm{m}$, with the EDX mapping analysis indicating the distribution of $\mathrm{Ti}, \mathrm{Al}, \mathrm{V}$ and $\mathrm{O}$ (as well as Au from the substrate). On the laser-nitrided samples, it was possible to find the edge of the drying stain where the suspension of wear debris had been dried out on Au. There were no particles which could be identified as containing any metallic species. However, the area of the drying stain showed $\mathrm{Na}, \mathrm{O}$ and some $\mathrm{C}$ containing particles.

\subsubsection{XPS analysis for the BM and TiN wear particles}

Fig. 10(a-b) provides the results of XPS analysis for the wear debris of the BM and laser-nitrided samples (dried onto $\mathrm{Au}$ ), including (a) survey scans for both the BM and laser-nitrided samples and (b) narrow scans only for the BM samples. Table 1 shows the composition (atom \%) of the elements detected in the wear debris of the BM and laser-nitrided samples.

In the suspension of the BM samples, there is a higher proportion of dried wear debris. Apart from the gold, it was possible to detect Ti, V, Al, O and C in the BM samples (in Table 1 and Fig. 10a). The $\mathrm{C}$ and a proportion of the $\mathrm{O}$ are from the inevitable atmospheric hydrocarbon contamination of airexposed samples. The significant findings in the XPS narrow scans of the BM samples (in Fig. 10b) can be summarised as follows: (i) The Ti $2 p_{3 / 2}-2 p_{1 / 2}$ doublet was fully consistent with the presence of $\mathrm{TiO}_{2}$, with the binding energy of the Ti $2 \mathrm{p}_{3 / 2}$ found at $459.0 \mathrm{eV}$. All the Ti detected was oxidised into this state; (ii) The $V 2 p_{3 / 2}$ peak was at $516.7 \mathrm{eV}$. This is exactly in the middle of the range for oxidised $V$, but due to the low levels present and consequently noisy spectrum it is not possible to state definitely whether this is in the $3+, 4+$ or $5+$ state; (iii) The Al $2 p$ peak cannot reliably be measured in the XPS study due to overlap with the very strong $\mathrm{Au} 4 \mathrm{f}$ signals. However, the $2 \mathrm{~s}$ peak measured at approximately $120 \mathrm{eV}$ indicated oxide, most likely $\mathrm{Al}_{2} \mathrm{O}_{3}$; (iv) The $\mathrm{O} 1 \mathrm{~s}$ peak showed a component at $530.6 \mathrm{eV}$. This is typical of metal oxide bonding and consistent with $\mathrm{TiO}_{2}$ and $\mathrm{Al}_{2} \mathrm{O}_{3}$. The other two components in the $\mathrm{O} 1 \mathrm{~s}$ peak are due to carbon bonding as $\mathrm{O}-\mathrm{C}$ and $\mathrm{O}=\mathrm{C}$ and are consistent with the minor components in the $C$ 1s peak. In summary, the wear debris in the BM samples is confirmed as being composed of oxidised materials from the constituent elements of the Ti6Al4V alloy: $\mathrm{Ti}, \mathrm{Al}$ and v. 
The amount of wear debris in the suspension of laser-nitrided samples was too low to be detected by XPS. The XPS results in Table 1 and Fig. 10(a) show only $\mathrm{Au}, \mathrm{C}, \mathrm{O}$ and $\mathrm{Na}$, in agreement with the SEMEDX results. This suggests a possible low level of sodium stearate contamination (i.e. soap) in the water of the wear test.

\subsubsection{Cell viability tests for the BM and TiN wear particles}

Fig. 11 provides the results of cell viability for the RAW 264.7 macrophages cultured with different concentrations $(10 \mu \mathrm{g}$ to $50 \mu \mathrm{g})$ of wear particles in the BM and laser-nitrided samples at two different time points ( $24 \mathrm{~h}$ and $48 \mathrm{~h}$ ). The particles were collected from the BM and laser-nitrided samples in the wear tests at two sliding distance $(200 \mathrm{~m}$ and $+800 \mathrm{~m})$. The OM images for the morphology of macrophages after $24 \mathrm{~h}$ and $48 \mathrm{~h}$ of culture (with the wear particles) are provided in Supplementary Information (Fig. S6 and S7).

A number of observations can be identified in the cell viability results in Fig. 11. Firstly, cell viability was higher in the laser-nitrided samples than the BM samples under all cell culture conditions (including different time points and concentration of wear particles). The average cell viability for the laser-nitrided samples (including the results of 200 and $+800 \mathrm{~m}$ sliding distance as well as the results of different concentration of wear particles) at $24 \mathrm{~h}$ and $48 \mathrm{~h}$ were $92.3 \%$ and $82.2 \%$, respectively, whereas the average cell viability for the BM samples at these time points were $81.4 \%$ and $69.5 \%$, respectively. It can also be concluded that there was great cell viability at $24 \mathrm{~h}$ than at $48 \mathrm{~h}$. Secondly, the difference in cell viability between the particles generated at 200 and $+800 \mathrm{~m}$ was negligible. This holds true for both the BM and laser-nitrided samples.

Thirdly, there is a decreasing trend in the viability of macrophages with increased concentration of wear particles (incl. both BM and laser-nitrided samples). Particularly, a simple linear relationship between the cell viability and concentration of wear particles can be observed in the BM samples. The cell viability at $24 \mathrm{~h}$ steadily decreased from $90.8 \%$ to $70.6 \%$ and the viability at $48 \mathrm{~h}$ also steadily decreased from $80.6 \%$ to $56 \%$ with increased concentration of wear particles from 10 to $50 \mu \mathrm{g}$. A different trend can be observed in the results of laser-nitrided samples. The cell viability at $24 \mathrm{~h}$ slightly decreased from $98.9 \%$ to $94.1 \%$ and at $48 \mathrm{~h}$ slightly decreased from $87.5 \%$ to $85.7 \%$ when the concentration increased from $10 \mu \mathrm{g}$ to $30 \mu \mathrm{g}$. It is important to note that the concentration of $40 \mu \mathrm{g}$ was identified as a critical point above which the reduction of cell viability was more pronounced. In $40 \mu \mathrm{g}$ concentration, the cell viability was found to be $87.9 \%$ and $79.0 \%$ at $24 \mathrm{~h}$ and $48 \mathrm{~h}$, respectively whilst it further dropped to $84.0 \%$ and $71 \%$, respectively with increased concentration to $50 \mu \mathrm{g}$.

To summarise the cell viability results, the wear particles from the laser-nitrided samples were confirmed to be less toxic compared with those from the BM samples.

\subsubsection{Biological reactions of macrophages in response to the BM and TiN wear particles}

Macrophages are multi-functional cells of the innate immune system, and their primary role is to maintain tissue homeostasis, including the (i) clearance of necrotic and apoptotic cellular debris, (ii) tissue remodelling following injury of the host and (iii) defence against foreign invaders [21].

A review conducted by Sridharan et al. [22] describes how cells of the innate immune system are the first to respond to the implantation of an implant in vascularized tissue. Shortly after the implantation, a layer of proteins, which are from the surrounding vasculature, immediately adsorb onto the implant surface, leading to the infiltration and adherence of cells including platelets, neutrophils, monocytes and macrophages. The adhered cells then release cytokines and chemokines that recruit tissue repair cells including fibroblasts and mesenchymal stem cells (MSC) to the injury site. They finally deposit a 
collagenous matrix and encapsulate the implant in a fibrous tissue layer. The process of collagen encapsulation is a hallmark of the so-called "Foreign Body Response" (FBR) [23].

It has been generally agreed that the development of periprosthetic osteolysis is highly related to wear debris generated continuously from an articulating surface of a hip implant. Wear debris can stimulate local and systemic biological reactions resulting in chronic inflammation, periprosthetic bone destruction, and finally implant loosening (and thus revision surgery is required) [21]. For the conventional metal-on-polyethylene (MoP) bearing surfaces, several hundreds of thousands of plastics particles may be generated during a single gait cycle [24]. The size of the plastics wear particles ranges between 0.1 and $10 \mu \mathrm{m}$ [25]. Metal-on-metal (MoM) hip implants usually produce smaller wear particles than those in the MoP prostheses.

The biological response of macrophages is dependent on the size and chemical composition of the wear particles. The macrophages can effectively phagocytose particles $<10 \mu \mathrm{m}$ [21], with particles of 0.1-1.0 $\mu \mathrm{m}$, which is the optimum size range for phagocytosis [26]. Regarding chemical composition, both the metal (Ti6Al4V) and plastics (polymethyl methacrylate and polyethylene) wear particles have been shown to stimulate macrophages to release many potent inflammatory mediators that induce bone resorption in vitro $[27,28,29]$. Neale et al. [30] reported that high concentrations of metal wear particles (Ti6Al4V, cp Ti, CoCr and 316L) can induce cell toxicity in long term culture.

The average size of BM wear particles in this study (i.e. between $1.4 \mu \mathrm{m}$ and $1.5 \mu \mathrm{m}$ ) was close to the aforementioned size range that is most efficiently phagocytosed. Taken together, the particle size for biological activity along with the potential toxicity of Ti6AI4V could be the reasons for the relatively high toxicity observed in the results of BM particles.

In comparison, a study conducted by Williams et al. [31] showed that the wear debris particles generated from different nitride materials including TiN, chromium nitride ( $\mathrm{CrN})$, and chromium carbon nitride ( $\mathrm{CrCN}$ ) showed reduced cytotoxic effect compared to the $\mathrm{CoCr}$ wear particles when cultured with U936 macrophages. TiN is known to be a biologically inert material and the reduced toxicity of TiN wear particles observed in this study can be attributive to this property.

\subsubsection{Potential toxicity of the TiN nano-particles}

It has been defined by the International Organization for Standardization that nano-particles (NPs) refer to structures whose sizes are in one, two, or three dimensions are within the range from $1 \mathrm{~nm}$ to $100 \mathrm{~nm}$ [32]. Nano-particles are always associated with toxicity concerns when used for diagnosis or treatment of human diseases. The sizes of the TiN particles are within the range of $1 \mathrm{~nm}$ to $100 \mathrm{~nm}$, and thus, they fulfil the definition of nano-particles. It is therefore essential to evaluate their toxicity to the macrophages which are at the frontline of the host defence system against pathogens (bacteria and viruses) as well as the foreign substances (wear particles).

It is generally accepted that toxicity is determined by the physical (size and shape) and chemical (surface charge and composition) characteristics as well as the presence or absence of a shell and active groups on the surface $[32,33]$. Hazards of nano-particles arise from their small size allowing them to penetrate through epithelial and endothelial barriers into the lymph and blood-and can be carried by the bloodstream and lymphatic system to different organs and tissues inside the human body. The toxicity induced can interfere with biological responses such as cell differentiation, protein synthesis as well as activation of pro-inflammatory genes and promote synthesis of inflammatory mediators [32]. The below discussion suggests possible explanations for the toxicity of TiN nanoparticles, based on existing knowledge of the mechanisms about nano-particle toxicity: 
Firstly, the size of the nano-particles is the main determinant of toxicity to the living organisms. Nanoparticles which are few nanometres in size are found to be more toxic than the nano-particles in a larger size $(>10 \mathrm{~nm}$ ) [34]. The size of TiN wear nano-particles were between $0.01 \mu \mathrm{m}$ and $0.04 \mu \mathrm{m}$ (or $10 \mathrm{~nm}$ and $40 \mathrm{~nm}$ ) which can be categorised as larger nano-particles in this sense. Secondly, the shapes of the nano-particles can influence their toxicity to the cells because they directly decide the extent of physical damage to the cell membrane [35] and influence the uptake into cells [36]. Nano-particles can shape as spheres, ellipsoids, cylinders, sheets, cubes, rods, etc. Reports in the literature are somewhat varied. However, spheroid particles tend to be less toxic compared with nano-tubes and nano-fibres [32]. This may go some way to explain why the TiN particles were found to be less toxic compared with the untreated BM particles (i.e. the irregular shape together with the higher aspect ratio causes more physical damage to the cells). Thirdly, the compositions of the nano-particles can affect their toxicity. TiN is reported to be bioinert. However, the results in the cell viability tests indicate that the TiN in a higher concentration (at $40 \mu \mathrm{g}$ or higher) could induce toxic effect to the cells. Further study is required to understand the possible cell damage mechanisms.

Finally, the toxicity of nano-particles can be related to the crystal structure of the surface oxides. Compared with the crystalline $\mathrm{TiO}_{2}$, amorphous $\mathrm{TiO}_{2}$ has more surface defects or active sites that are capable of causing the formation of reactive oxygen species (ROS) which are short-lived and highly reactive molecules. Excessive cellular levels of ROS can cause damage to proteins, nucleic acids, lipids, membranes and organelles, leading to apoptosis [37]. It has been reported by the authors' previous study [9] that the surfaces after laser treatment were in the crystalline form. Since the particles were generated from the surfaces, the oxides on the particles are very likely to bear the same crystal structure as their parent surfaces. This may be one of the reasons explaining the lesser toxicity found in the TiN particles. Nitrides also exist in the surfaces of TiN particles. The presence of nitrides themselves in the surfaces and their crystal structure could contribute to the reduced toxicity, as TiN is known to be a biocompatible material [38]. However, it is still inconclusive to confirm the roles of surface nitrides in reducing toxicity of the TiN particles. Further research is therefore required.

\subsubsection{Antibacterial properties of the BM and TiN wear particles}

Both the laser-nitrided (or TiN) and BM wear particles exhibited antibacterial activity against $E$. coli, showing a significant difference $(p<0.0001)$ relative to the control. As illustrated in Fig. 12 the lasernitrided and BM wear particles exhibited similar logarithmic reductions in the number of CFU/mL (log reductions of $0.68(79.1 \%)$, and 0.67 (78.6\%) respectively). This indicates that against $E$. coli, wear particles from materials display a slight antimicrobial effect.

In relation to $S$. aureus it is evident that only wear particles derived from laser-nitrided Ti exhibited antibacterial activity, displaying a log reduction of $0.83(85.2 \%)$ relative to the control $(p<0.0001)$. Interestingly, no antimicrobial effect was seen with the BM samples, suggesting that these Grampositive bacteria are more susceptible to wear particles generated after the laser nitriding process.

These observations highlight that wear debris, which can often pose a risk for orthopaedic implant success [39], may demonstrate beneficial antibacterial activity against two of the most commonly implicated pathogens in orthopaedic implant infection, especially when laser-nitriding is performed on the Ti surface [40]. The contrast in antimicrobial activity between the two species studied could be explained by the differences in cell wall structure between Gram-negative (E. Coli) and Gram-positive (S. aureus) bacteria, and this may confer differences in the susceptibility of each microorganism to the effects of TiN particulates. However, many studies published in the literature, although largely based on particles composed of silver, have found a trend for Gram-positive species, with their thicker cell wall, to show greater resistance to particulate metals [41,42]. This trend is in contrast to our current 
findings based on the TiN wear particles, in relation to S. aureus. Both intrinsic (bacterial-derived) and extrinsic (due to physical and chemical properties of the material, including processing conditions), can have an influence antimicrobial effect. Cell wall electronegativity, particulate internalisation, the generation of reactive oxygen species (ROS), and genetically-mediated resistance to metal ions may all play a role [43]. Further work is warranted to investigate the mechanisms that underpin bacterial cell/wear particle interactions, and the effects of laser-nitriding.

Research has shown that the antibacterial activity of nano-particles can be influenced by both the particle's size and shape [44]. Smaller nano-particles have larger specific surface areas which will result in greater interaction with the bacterial cell, and greater likelihood of internalisation of the particle through the cell membrane, or penetration into the biofilm structure. It is also known that the particle shape is an important factor in antimicrobial activity of nano-particles. Nano-particles with different geometries can cause varying degrees of bacterial cell damage through interactions with periplasmic enzymes [44]. The shape effect of antibacterial activity is related to specific surface area and facet reactivity (e.g. rod-like nano-particles are more effective than spherical nano-particles) [44]. The antibacterial effect of TiN nano-particles against $\mathrm{S}$. aureus could be attributed to their ellipsoid shape. However, this finding is still preliminary and a further controlled study which compares the TiN nanoparticles in different shape is required to verity this. 


\subsection{Conclusions}

The Ti6Al4V alloy was laser-nitrided in the open-air using fibre laser in modulated mode. The duty cycle was varied between $60 \%$ (pulsed mode) and $100 \%$ (CW mode). The main purpose of applying laser nitriding is to improve the wear resistance of Ti6Al4V in order to minimise the generation of wear particles from the surfaces. Both the laser-nitrided surfaces and the wear particles were carefully studied and analysed.

The important findings in relation to the laser-nitrided surfaces:

1) Laser nitriding performed at the duty cycle of $60 \%$ was found to be optimal, resulting in a homogenous, gold-coloured surface with no cracks.

2) The LNZ depth increased from $41 \mu \mathrm{m}$ to $205 \mu \mathrm{m}$ when increasing the duty cycle from $60 \%$ to $100 \%$.

3) The hardness in LNZ lay between $795 \mathrm{HV}$ and $844 \mathrm{HV}$ and was much higher than in the HAZ or the BM.

4) The XRD results showed the existence of $\alpha-\mathrm{Ti}, \beta-\mathrm{Ti}$ and TiN in the nitrided surfaces whilst only $\alpha$-Ti was present in the BM surface.

5) The nitrides formed at higher duty cycles (DC80 and DC100) were more brittle compared with that treated at the lower duty cycle (DC60).

The important findings in relation to the wear particles:

1) The average size of wear particles from the laser-nitrided surfaces was smaller than those from the BM surfaces $(0.01 \mu \mathrm{m}$ to $0.04 \mu \mathrm{m}$ versus $1.5 \mu \mathrm{m})$.

2) The wear particles from the laser-nitrided surfaces were confirmed to be less toxic towards RAW264.7 macrophages, yet displayed antimicrobial properties against S. aureus, compared with those from the BM surfaces.

The antibacterial activity of TiN wear nano-particles against $S$. aureus can be attributed to their very small size $(0.01 \mu \mathrm{m}$ to $0.04 \mu \mathrm{m})$ whereas the particles from the BM surfaces had a significantly larger size $(1.5 \mu \mathrm{m})$

\section{Acknowledgement}

The work described in this paper was supported by research grants from the Queen's University Belfast, awarded to C-WC and LC (D8201MAS, D8304PMY). 


\section{References}

[1] US Food \& Drug Administration (FDA). (2019a). General Information about Hip Implants [Online]. Available at https://www.fda.gov/medical-devices/metal-metal-hip-implants/generalinformation-about-hip-implants (Accessed 21/9/2020).

[2] US Food \& Drug Administration (FDA). (2019b). Concerns about Metal-on-Metal Hip Implants [Online]. Available at https://www.fda.gov/medical-devices/metal-metal-hip-implants/concernsabout-metal-metal-hip-implants (Accessed 21/9/2020).

[3] Drummond, J., Tran, P., Fary, C., Metal-on-metal hip arthroplasty: a review of adverse reactions and patient management, Journal of Functional Biomaterials. 6(3) (2015) 486-99.

[4] Green, B., Griffiths, E., Almond, S., Neuropsychiatric symptoms following metal-on-metal implant failure with cobalt and chromium toxicity, BMC Psychiatry. 17(1) (2017) 33.

[5] Gomes, C.C., Moreira, L.M., Santos, V.J., Ramos, A.S., Lyon, J.P., Soares, C.P., Santos, F.V., Assessment of the genetic risks of a metallic alloy used in medical implants, Genetics and Molecular Biology. 34(1) (2011) 116-121.

[6] van Hove, R.P., Sierevelt, I.N., van Royen, B.J., Nolte, P.A., Titanium-nitride coating of orthopaedic implants: a review of the literature, BioMed Research International. 2015 (2015) 485975.

[7] Chan, C.W., Lee, S., Smith, G., Sarri, G., Ng, C.H., Sharba, A., Man, H.C., Enhancement of wear and corrosion resistance of beta titanium alloy by laser gas alloying with nitrogen, Applied Surface Science. 367 (2016) 80-90.

[8] Chan, C.W., Lee, S., Smith, G.C., Donaghy, C., Fibre laser nitriding of titanium and its alloy in open atmosphere for orthopaedic implant applications: Investigations on surface quality, microstructure and tribological properties, Surface and Coatings Technology. 309 (2017a) 628-640.

[9] Chan, C.W., Carson, L., Smith, G.C., Morelli, A., Lee, S., Enhancing the antibacterial performance of orthopaedic implant materials by fibre laser surface engineering, Applied Surface Science. 404 (2017b) 67-81.

[10]Chan, C.W., Carson, L., Smith, G.C., Fibre laser treatment of martensitic NiTi alloys for load-bearing implant applications: Effects of surface chemistry on inhibiting Staphylococcus aureus biofilm formation, Surface and Coatings Technology, 349 (2018) 488-502.

[11]Dion, I., Baquey, C., Candelon, B., Monties, J.R., Hemocompatibility of titanium nitride, International Journal of Artificial Organs, 15(10) (1992) 617-621.

[12]Datta, S., Das, M., V. Balla, Bodhak, S., Murugesan, V., Mechanical, wear, corrosion and biological properties of arc deposited titanium nitride coatings, Surface and Coatings Technology. 344 (2018) 214-222.

[13]Gordin, D.M., Gloriant, T., Chane-Pane, V., Busardo, D., Mitran, V., Höche, D., Vasilescu, C., Drob, S.I., Cimpean, A., Surface characterization and biocompatibility of titanium alloys implanted with nitrogen by Hardion+ technology, Journal of Materials Science: Materials in Medicine. 23(12) (2012) 2953-2966.

[14]Brunello, G., Brun, P., Gardin, C., Ferroni, L., Bressan, E., Meneghello, R., Zavan, B., Sivolella, S., Biocompatibility and antibacterial properties of zirconium nitride coating on titanium abutments: An in vitro study, Journal of Oral Implantology. 13(6) (2018) 0199591.

[15]Annunziata, M., Oliva, A., Basile, M., Giordano, M., Mazzola, N., Rizzo, A., Lanza, A., Guida, L., The effects of titanium nitride-coating on the topographic and biological features of TPS implant surfaces, Journal of Dentistry. 39(11) (2011) 720-728.

[16]Chan, C.W., Chang, X., Bozorgzadeh, M.A., Smith, G.C., Lee, S., A single parameter approach to enhance the microstructural and mechanical properties of beta Ti-Nb alloy via open-air fiber laser nitriding, Surface and Coatings Technology. 383 (2020) 125269. 
[17]Miles, A. A., Misra, S. S., Irwin, J. O., The estimation of the bactericidal power of the blood, The Journal of Hygiene. 38(6) (1938) 732-749.

[18]Abboud, J.H., Effect of processing parameters on titanium nitrided surface layers produced by laser gas nitriding, Surface Coating Technology. 214 (2013) 19-29.

[19]Donaghy, C., McFadden, R., Kelaini, S., Carson, L., Margariti, A., Chan, C.W., Creating an antibacterial surface on beta TNZT alloys for hip implant applications by laser nitriding, Optics and Laser Technology. 121 (2020) 105793.

[20]Chang, X., Smith, G. C., Quinn, J., Carson, L., Chan, C. W., Lee, S., Optimization of anti-wear and anti-bacterial properties of beta TiNb alloy via controlling duty cycle in open-air laser nitriding, Journal of the Mechanical Behavior of Biomedical Materials. 110 (2020) 103913.

[21]Nich, C., Takakubo, Y., Pajarinen, J., Ainola, M., Salem, A., Sillat, T., et al. Macrophages - key cells in the response to wear debris from joint replacements, Journal of Biomedical Materials Research A. 101(10) (2013) 3033-3045.

[22]Sridharan, R., Cameron, A.R., Kelly, D.J., Kearney, C.J., O'Brien, F.J., Biomaterial based modulation of macrophage polarization: a review and suggested design principles, Materials Today. 18(6) (2015) 313-325.

[23]Anderson, J. M., Rodriguez, A., \& Chang, D. T. (2008). Foreign body reaction to biomaterials, Seminars in immunology. 20(2), 86-100.

[24]McKellop, H.A., Campbell, P., Park, S.H., Schmalzried, T.P., Grigoris, P., Amstutz, H.C., Sarmiento, A., The origin of submicron polyethylene wear debris in total hip arthroplasty, Clinical Orthopaedics and Related Research. 311 (1995) 3-20.

[25]Nine, M., Choudhury, D., Hee, A., Mootanah, R., Osman, N., Wear debris characterization and corresponding biological response: artificial hip and knee joints, Materials. 7(2) (2014) 980-1016.

[26] Hatton, A., Nevelos, J.E., Matthews, J.B., Fisher, J., Ingham, E., Effects of clinically relevant alumina ceramic wear particles on TNF- $\alpha$ production by human peripheral blood mononuclear phagocytes, Biomaterials. 24(7) (2003) 1193-1204.

[27]Haynes, D.R., Rogers. S.D., Hay, S., Pearcy, M.J., Howie, D.W., The differences in toxicity and release of bone-resorbing mediators induced by titanium and cobalt-chromium-alloy wear particles, Journal of Bone and Joint Surgery. 75(6) (1993) 825-834.

[28]Shanbhag, A.S., Jacobs, J.J., Black, J., Galante, J.O., Glant, T.T., Human monocyte response to particulate biomaterials generated in vivo and in vitro, Journal of Orthopaedic Research. 13(5) (1995) 792-801.

[29]Horowitz, S.M., Gonzales. J.B., Effects of polyethylene on macrophages, Journal of Orthopaedic Research. 15(1) (1997) 50-56.

[30]Neale, S.D., Haynes, D.R., Howie, D.W., Murray, D.W., Athanasou, N.A., The effect of particle phagocytosis and metallic wear particles on osteoclast formation and bone resorption in vitro, Journal of Arthroplasty. 15 (2000) 654-662.

[31]Williams, S., Tipper, J.L., Ingham, E., Stone, M.H., Fisher, J., In vitro analysis of the wear, wear debris and biological activity of surface-engineered coatings for use in metal-on-metal total hip replacements, The Proceedings of the Institution of Mechanical Engineers H. 217 (2003) 155-163.

[32]Sukhanova, A., Bozrova, S., Sokolov, P., Berestovoy, M., Karaulov, A., Nabiev, I., Dependence of nanoparticle toxicity on their physical and chemical properties, Nanoscale Research Letter. 13 (2018) 44.

[33]Barcelo, D., Farre, M. (2012) Analysis and Risk of Nanomaterials in Environmental and Food Samples (1 $1^{\text {st }}$ ed.), vol. 59, Elsevier.

[34]Huo, S., Jin, S., Ma, X., Xue, X., Yang, K., Kumar A., et al. Ultra small gold nanoparticles as carriers for nucleus-based gene therapy due to size dependent nuclear entry, ACS Nano. 8(6) (2014) 5852 5862. 
[35]Hu, W., Peng, C., Lv M, Li X, Zhang, Y., Chen, N., et al. Protein corona mediated mitigation of cytotoxicity of graphene oxide, ACS Nano. 5(5) (2011) 3693-3700.

[36]Albanese, A., Tang, P.S., Chan, W.C., The effect of nanoparticle size, shape, and surface chemistry on biological systems, Annual Review Biomedical Engineering. 14 (2012) 1-16.

[37]Redza-Dutordoir, M., Averill-Bates, D.A., Activation of apoptosis signalling pathways by reactive oxygen species, Biochimica et Biophysica Acta (BBA) - Molecular Cell Research. 1863(12) (2016) 2977-2992.

[38]Sovak, G., Weiss, A., Gotman, I., Osseointegration of Ti6Al4V alloy implants coated with titanium nitride by a new method, The Journal of bone and joint surgery. British volume. 82 (2000) 290296.

[39]Ribeiro, M., Monteiro, F.J., Ferraz, M.P., Infection of orthopedic implants with emphasis on bacterial adhesion process and techniques used in studying bacterial-material interactions, Biomatter. 2(4) (2012) 176-194.

[40]Keegan, G.M., Learmonth, I.D., Case. C.P., Orthopaedic metals and their potential toxicity in the arthroplasty patient: A review of current knowledge and future strategies, The Journal of bone and joint surgery. British volume. 89 (2007) 567-573.

[41]Feng, Q.L., Wu, J., Chen, G.Q., Cui, F.Z., Kim, T.N., Kim, J.O., A mechanistic study of the antibacterial effect of silver ions on Escherichia coli and Staphylococcus aureus, Journal of Biomedical Materials Research. 52 (2000) 662-668.

[42]Mukha, I.P., Eremenko, A.M., Smirnova, N.P., Mikhienkova, A.I., Korchak, G.I., Gorchev, V.F., et al. Antimicrobial activity of stable silver nanoparticles of a certain size, Applied Biochemistry and Microbiology. 49 (2013) 199-206.

[43]Slavin, Y.N., Asnis, J., Häfeli, U.O., Bach, H., Metal nanoparticles: understanding the mechanisms behind antibacterial activity, Journal of Nanobiotechnology. 15(1) (2017) 65.

[44]Wang, L., Hu, C., Shao, L., The antimicrobial activity of nanoparticles: present situation and prospects for the future, International Journal of Nanomedicine. 12 (2017) 1227-1249. 